\title{
FABRICAÇÃO DIGITAL PARA AUXILIAR NO ENSINO-APRENDIZADO DE ALUNOS COM DEFICIÊNCIA VISUAL: ESTUDO DE CASO DOS SISTEMAS NANOESTRUTURADOS
}

Mariana Pohlmann

Universidade Federal do Rio Grande do Sul marianapohlmann@gmail.com

Wagner Soares Rossi

Universidade Federal do Rio Grande do Sul wsrossi@gmail.com
Clariana Fischer Brendler

Universidade Federal do Rio Grande do Sul clariana.brendler@ufrgs.br

Fábio Gonçalves Teixeira

Universidade Federal do Rio Grande do Sul fabiogt@ufrgs.br

Wilson Kindlein Júnior

Universidade Federal do Rio Grande do Sul wilsonkindleinjunior@gmail.com

Resumo: Dados obtidos com o Censo Demográfico, em 2010, revelaram que quase $24 \%$ da população brasileira possuem algum tipo de deficiência, sendo a deficiência visual a de maior ocorrência. Dentre as políticas sociais que buscam atender às diferentes necessidades dos cidadãos, vale destacar o direito universal à educação. Recursos didáticos de diferentes naturezas vêm sendo empregados como ferramenta para auxiliar o ensinoaprendizagem de pessoas com deficiência visual. Vale, porém, questionar se esses recursos são realmente eficazes na compreensão de determinados conteúdos? Assim, o presente trabalho propõe a validação de modelos didáticos 3D para permitir a compreensão de sistemas nanoestruturados por deficientes visuais. A avaliação dos modelos foi realizada por usuário cego e, para tanto, foi utilizada uma adaptação do instrumento SETT (Alunos, Ambiente, Tarefas e Ferramentas). Os resultados indicam que os modelos produzidos por impressão 3D necessitam de revisão no que tange à legenda em Braille, mas, quanto aos demais aspectos, são viáveis para o uso como recurso didático para alunos com deficiência visual.

Palavras-chave: sistemas nanoestruturados, deficiência visual, modelos didáticos táteis, fabricação 3D, design centrado no usuário.

Abstract: Obtained data from Demographic Census in 2010 revealed that nearly $24 \%$ of the Brazilian population has some kind of disability, and visual impairment is the greater occurrence. Among the social policies that seek to meet the different needs of citizens, it is worth highlighting the universal right to education. Teaching resources of different nature have been used as tool to assist the teaching-learning of people with visual impairment. It worth, however, to ask whether these features are really effective in understanding certain content? Thus, this paper proposes the 
validation of educational 3D models to enable visually impaired people to understand the nanostructured systems. The evaluation of the models was performed by blind user and an adaptation of the instrument SETT (Students, Environment, Tasks and Tools) was used as method. The results indicate that the models produced by $3 D$ printing need revision regarding the Braille subtitles, but regarding other aspects, are feasible for use as a teaching resource for students with visual impairment.

Keywords: nanostructured systems, visual impairment, tactile didactic models, 3D manufacturing, user-centered design.

\section{INTRODUÇÃO}

Apesar de a Constituição Brasileira declarar que, independentemente das condições dos cidadãos, todos têm os mesmo direitos, as pessoas com deficiência nem sempre conseguem clamar por seus direitos. Essa barreira ocorre "devido a desvantagens impostas pela restrição de funcionalidades e pela sociedade, que thes impõe barreiras físicas, legais e de atitude" (OLIVEIRA, 2012).

Para ultrapassar essa barreira, percebe-se um aumento da mobilização social para atender às diferentes necessidades das pessoas nas últimas décadas. Para Bruno e Mota (2001) o modelo social da deficiência se fortalece como um processo bilateral cuja participação ativa das pessoas e do sistema tem como objetivo comum à formação de uma sociedade para todos. Dentre as políticas sociais, destaca-se o direito à educação das pessoas com deficiência. A educação é um processo fundamental ao desenvolvimento do ser humano e, portanto, deve ser priorizada para que o sujeito se aproprie da história e da cultura, estabelecendo as instituições de ensino como um espaço de acesso ao saber (BATISTA; MENEZES, 2008).

No caso de pessoas com deficiência visual, já é bem estabelecida a utilização de recursos didáticos como livros em Braille, sistemas de ampliação de letras e softwares com sintetizador de voz que fazem a leitura de textos na tela do microcomputador. Esses indivíduos têm o sentido do tato muito desenvolvido. Portanto, objetos com diferentes tamanhos e texturas proporcionam sensações capazes de instigar a formação de imagens mentais importantes para comunicação, estética e formação de conceitos (SÁ; CAMPOS; SILVA, 2007). Entretanto, os recursos supracitados não são eficazes no ensino-aprendizado de determinados conteúdos, tais como estruturas nanométricas.

Neste sentido, o presente trabalho tem como objetivo a validação de modelos de sistemas nanoestruturados desenvolvidos previamente por Pohlmann e colaboradores (2015). Os modelos do nanolipossoma, do carreador lipídico nanoestruturado, da nanoesfera e da nanocápsula foram avaliados por usuário com deficiência visual. Suas considerações acerca do tamanho, elementos e materiais poderão ser utilizadas como diretrizes na produção de outros modelos didáticos.

\section{PRODUÇÃO DOS MODELOS DIDÁTICOS DOS SISTEMAS NANOESTRUTURADOS}

No estudo publicado por Pohlmann e colaboradores (2015) a produção dos modelos dos sistemas nanoestruturados percorreu quatro etapas: (i) entendimento e conceituação da deficiência visual, aspectos ligados à educação inclusiva e critérios 
para a confecção de recursos didáticos; (ii) desenho e estilização das imagens dos sistemas nanoestruturados; (iii) modelagem virtual tridimensional dos sistemas e; (iv) impressão tridimensional dos sistemas.

\subsection{Educação e modelos didáticos para usuários com deficiência visual}

No Brasil, a Fundação Dorina Nowill é a instituição pioneira no ensino integrado de alunos com deficiência visual. Independentemente do nível da deficiência visual (seja cegueira ou baixa visão), para viabilizar esse ensino integrado, torna-se necessário um esforço conjunto na capacitação de recursos humanos e da adaptação das práticas pedagógicas (CRÓs et al., 2006).

Os indivíduos com deficiência visual fazem de seu sistema háptico a interface com os objetos e o ambiente ao seu redor. Sendo assim, a elaboração de modelos didáticos deve obedecer a certos critérios de produção já que estão constantemente em contato com o aluno. Quanto à seleção dos materiais, deve-se dar preferência àqueles aprazíveis ao tato, que não provoquem irritação cutânea e que ofereçam resistência suficiente; já que os modelos serão intensamente manuseados. Com relação ao uso, a produção dos modelos deve ser farta para que vários alunos sejam atendidos simultaneamente; deve haver ampla variedade de modelos para que esses possibilitem a diversidade de experiências; bem como atender aspectos de percepção tátil e/ou visual (no caso de baixa visão) para que o estímulo seja significativo (CERQUEIRA; FERREIRA, 2000; SÁ; CAMPOS; SILVA, 2007).

\subsection{Desenho e estilização da forma dos sistemas nanoestruturados}

A nanotecnologia consiste no desenvolvimento, caracterização e aplicação de materiais em escala nanométrica (MIHRANYAN; FERRAZ; STRØMME, 2012; MORITZ; GESKE-MORITZ, 2013). Até o presente momento, pesquisadores da área de química reconhecem diversos nanossistemas que se diferenciam de acordo com sua composição. Devido à alta complexidade e da tênue diferenciação entre as estruturas, faz-se necessária a elaboração de desenhos esquemáticos para esclarecer a diferença entre elas.

Dentre os sistemas reconhecidos, destacam-se os nanolipossomas, as nanoemulsões e as nanopartículas. Os nanolipossomas (Figura 1a) têm estruturas de membranas lipídicas em bicamada compostas de fosfolipídios. As nanoemulsões (Figura 1b) são dispersões compostas por dois líquidos imiscíveis, como óleo e água (O/A). As nanopartículas podem ser subdivididas em nanopartículas lipídicas sólidas (Figura $1 \mathrm{C})$, semelhantes às nanoemulsões $(\mathrm{O} / \mathrm{A})$, mas o óleo é substituído por um lipídeo sólido e; carreadores lipídicos nanoestruturados (Figura 1d), os quais são produzidos a partir da mistura entre lipídios sólidos e lipídios líquidos, tais como as manteigas; nanocápsulas (Figura 1e), nas quais o invólucro polimérico está disposto ao redor de um núcleo oleoso; e nanoesferas (Figura 1f), formadas por uma matriz polimérica (SCHAFFAZICK et al., 2003; SILVA, 2004; MÜLLER, 2007; KHAN et al., 2012; WANG et al., 2014). 
a

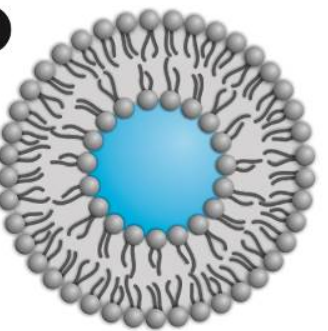

d

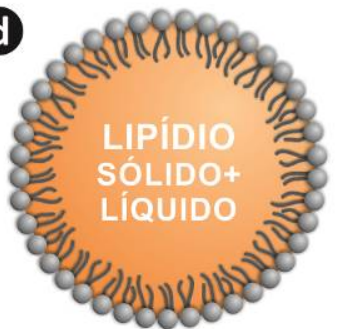

b

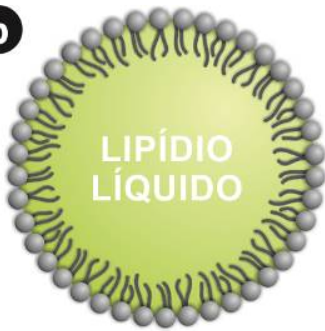

e

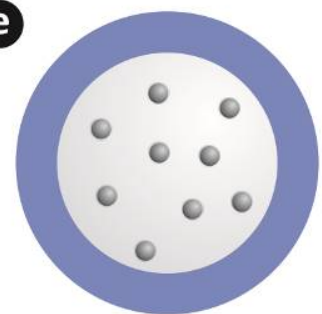

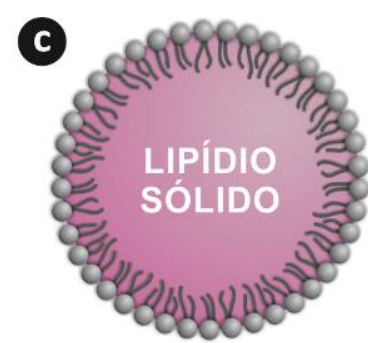

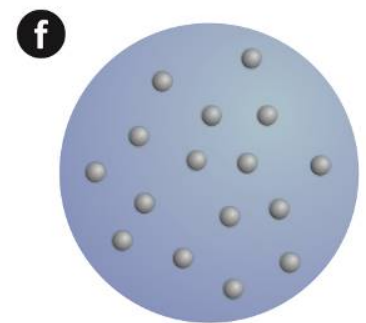

Figura 1 - Desenho esquemático dos sistemas nanoestruturados: (a) nanolipossoma; (b) nanoemulsão; (c) nanopartícula lipídica sólida; (d) carreador lipídico nanoestruturado; (e) nanocápsula e; (f) nanoesfera.

Modificado de Schaffazick et al., 2003; Wang et al., 2014 e Pohlmann et al., 2015.

\subsection{Modelagem virtual 3D}

Os modelos virtuais 3D foram criados a partir de uma semiesfera (Figura 2). Em substituição ao preenchimento gradiente, volumes foram empregados a fim de primar os aspectos táteis da representação. Para a indicação dos diferentes estados da matéria (sólido e líquido), inscrições em Braille foram acrescentadas sobre as superfícies dos núcleos da nanoemulsão (Figura 2b), da nanopartícula lipídica sólida (Figura 2c) e do carreador lipídico nanoestruturado (Figura 2d).
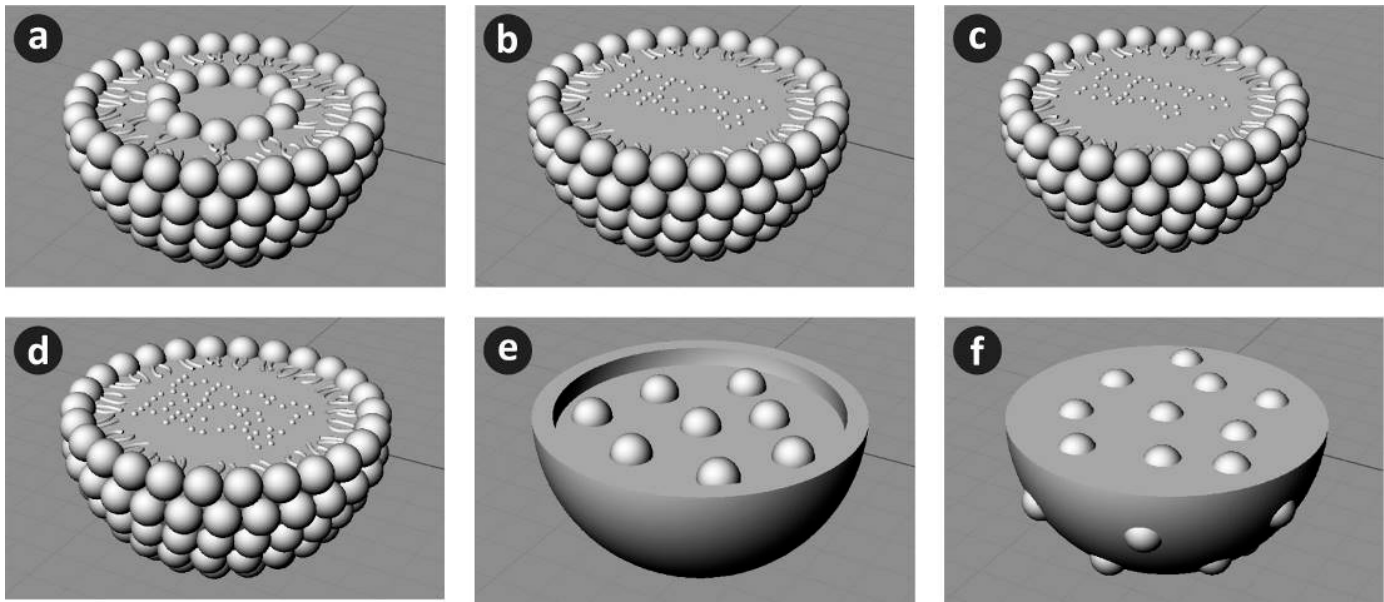

Figura 2 - Modelagem virtual 3D dos sistemas nanoestruturados: (a) nanolipossoma; (b) nanoemulsão; (c) nanopartícula lipídica sólida; (d) carreador lipídico nanoestruturado; (e) nanocápsula e; (f) nanoesfera.

Modificado de Pohlmann et al., 2015. 


\subsection{Impressão 3D}

O modelo didático do nanolipossoma foi produzido pela impressora modelo Objet30 Pro, marca Stratasys, que imprime em resina translúcida e utiliza a tecnologia PolyJet (semelhante à jato de tinta). Já os modelos do carreador lipídico nanoestruturado, da nanoesfera e da nanocápsula foram obtidos pela impressora modelo UP Plus 2, da marca Sintetize 3D. Este equipamento utiliza a tecnologia FDM (Deposição de Material Fundido, do inglês Fused Deposition Modeling) e o material conhecido como PLA (ácido polilático).

A partir dos modelos didáticos obtidos por impressão 3D, foi possível realizar a avaliação pelo usuário.

\section{AVALIAÇÃO PELO USUÁRIO}

A avaliação pelo usuário é uma etapa de fundamental importância no desenvolvimento de produtos centrados no usuário. Este feedback visa garantir a eficácia do produto. Para a realização do presente estudo, esta etapa foi realizada, mesmo sem utilizar todas as premissas, com o auxílio do instrumento SETT (Alunos, Ambiente, Tarefas e Ferramentas; do inglês, Student, Environment, Task, Tools) desenvolvido por Zabala (1998). O autor considera como aspectos inerentes deste instrumento (i) as necessidades dos alunos, incluindo suas deficiências e habilidades; (ii) as características do ambiente, incluindo recursos didáticos disponíveis, disposição física e tecnologias e iluminação; (iii) as tarefas realizadas pelos alunos no ambiente e; (iv) as ferramentas e tecnologias que podem auxiliar o aluno no desempenho das tarefas. Além disso, as questões a serem respondidas são:

- O tamanho dos recursos didático está adequado?

- Você consegue identificar os elementos que compõem este recurso?

- O material tem um toque agradável ou machuca?

- Este recurso auxilia na compreensão do conteúdo?

A avaliação foi realizada por um participante do Programa Incluir UFRGS ${ }^{1}$ com quatro modelos produzidos: nanolipossoma (Figura 3a), carreador lipídico nanoestruturado (Figura 3b), nanoesfera (Figura 3c) e nanocápsula (Figura 3d). As perguntas foram feitas à medida que o usuário experimentava os modelos didáticos.

\footnotetext{
${ }^{1}$ O Programa Incluir UFRGS foi desenvolvido pela Secretaria de Ensino Superior e pela Secretaria de Educação Especial do MEC e apoia "estratégias de inclusão, acessibilidade e permanência de pessoas com deficiência, com e para a comunidade universitária, no âmbito do ensino, pesquisa, extensão e gestão administrativa" (UFRGS, 2016).
} 


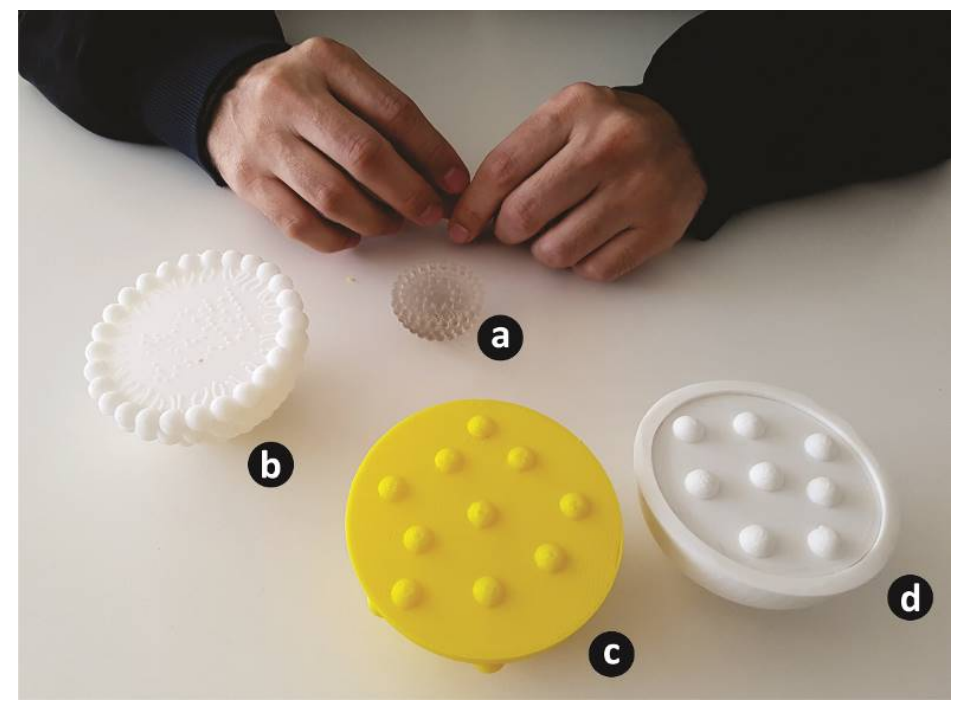

Figura 3 - Avaliação pelo usuário dos modelos didáticos do (a) nanolipossoma, (b) carreador lipídico nanoestruturado, (c) nanoesfera e (d) nanocápsula.

Fonte: Elaborado pelo autor, com base na pesquisa realizada.

Acerca do tamanho, o usuário considerou satisfatórios os modelos do carreador lipídico nanoestruturado, da nanoesfera e da nanocápsula. Já o modelo do nanolipossoma possui dimensões demasiadamente pequenas (Figura 4a), dificultando sua manipulação. Quanto à identificação dos elementos que compõem as estruturas, com exceção do nanolipossoma, não houve queixa por parte do usuário. Entretanto, as legendas escritas com sistema Braille devem revisadas para que haja uniformidade na altura e espaçamento dos caracteres (Figura 4b).
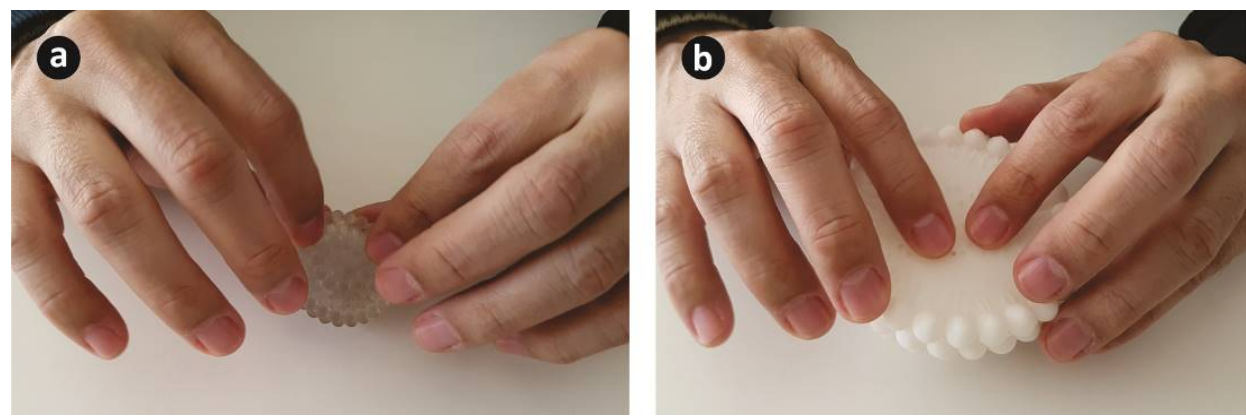

Figura 4 - Avaliação dos modelos didáticos: usuário tentando (a) identificar os elementos do nanolipossoma e (b) tentando ler a legenda no sistema Braille.

Fonte: Elaborado pelo autor, com base na pesquisa realizada.

No que tange os materiais utilizados na produção dos modelos didáticos, o usuário reconheceu a qualidade superior do nanolipossoma (impresso em resina pela tecnologia Polyjet), mas ressaltou que ambos os materiais e processos de impressão cumprem seu propósito de elucidação das nanoestruturas. Como o usuário não nasceu com a deficiência visual, pôde conhecer algumas ilustrações de livros didáticos utilizados em disciplinas da área das ciências. Ele relatou que os modelos propostos têm potencial para facilitar a compreensão do conteúdo e estruturas expostas tanto por alunos com deficiência visual, quanto para os videntes. 


\section{CONSIDERAÇÕES FINAIS}

Os modelos didáticos de sistemas nanoestruturados foram desenvolvidos por Pohlmann e colaboradores (2015) a fim de serem utilizados como ferramenta de ensino tanto para alunos videntes quanto para aqueles com deficiência visual, seja cegueira ou baixa visão. A utilização dos modelos 3D realizada no presente trabalho, através da avaliação dos modelos por usuário cego, auxiliou na elucidação de aspectos a serem levados em consideração no que tange à produção desses recursos.

De maneira geral, ambas as técnicas de impressão 3D são viáveis para a obtenção dos modelos de sistemas nanoestruturados. Além disso, os materiais empregados não interferem na interpretação das estruturas que compõem os modelos. Já o tamanho utilizado pode prejudicar a compreensão de detalhes das nanoestruturas. Quanto às legendas em Braille, estas devem passar por uma revisão para que se obtenha mais uniformidade entre os caracteres. Sendo assim, este estudo indica a viabilidade da utilização da fabricação digital 3D como tecnologia para obtenção de modelos didáticos, os quais podem ser estendidos a outros conteúdos da ciência e, até mesmo, a outras disciplinas.

\section{AGRADECIMENTOS}

Ao Conselho Nacional de Desenvolvimento Científico e Tecnológico - CNPq e à Coordenação de Aperfeiçoamento de Pessoal de Nível Superior - CAPES (Processo BEX 5786/15-0) pelo apoio aos autores deste artigo. Ao Centro Multiusuário de Prototipagem Rápida (CMPR) do Parque Científico e Tecnológico da UFRGS. Agradecemos, também, ao Programa Incluir UFRGS.

\section{REFERÊNCIAS}

BATISTA, MLFS; MENEZES, MS. O Design Gráfico e o Design Instrucional na Educação a Distância. In: Jofre Silva (org.). Design, Arte e Tecnologia 4. 4ed.São Paulo, SP: Rosari, 2008 , v. 1, p. 01-15.

BRUNO, MMG; MOTA, MGB. Programa de capacitação de recursos humanos do ensino fundamental: deficiência visual. Brasília: Ministério da Educação, Secretaria de Educação Especial, 2001.

CERQUEIRA, JB; FERREIRA, MA. Os recursos didáticos na educação especial. In: Revista Benjamin Constant. Rio de Janeiro, 15ed., 2000.

CRÓS, CX et al. Classificações da deficiência visual: compreendendo conceitos esportivos, educacionais, médicos e legais. In: Revista Digital. Buenos Aires, n. 93, 2006.

KHAN, AW et al. Potentials and challenges in self nanoemulsifying drug delivery systems. In: Expert Opin Drug Deliv., n. 9, 2012. p. 1305-17.

MIHRANYAN, A; FERRAZ, N; STRØMME, M. Current status and future prospects of nanotechnology in cosmetics. In: Progress in Materials Science, n. 57, 2012. p. 875910.

MORITZ, M; GESKE-MORITZ, M, The newest achievements in synthesis, immobilization and practical applications of antibacterial nanoparticles. In: Chemical Engineering Journal, n. 228, 2013. p. 596-613. 
MÜLLER, RH. Lipid nanoparticles: recent advances. In: Adv. Drug Deliv. n. 59, 2007. p. 375-376.

OLIVEIRA, LMB. Cartilha do Censo 2010 - Pessoas com Deficiência. Secretaria de Direitos Humanos da Presidência da República / Secretaria Nacional de Promoção dos Direitos da Pessoa com Deficiência / Coordenação-Geral do Sistema de Informações sobre a Pessoa com Deficiência; Brasília: SDH-PR/SNPD, 2012.

POHLMANN, $M$ et al. Drawing, virtual modeling and 3D print in the production of didactic models for the teaching-learning of visually impaired students: case study of nanostructured systems. In: International Journal of Education and Research, v. 3, n. 12, 2015. p. 453-466.

SÁ, ED; CAMPOS, IM; SILVA, MBC. Atendimento educacional especializado: deficiência visual. Brasília: Ministério da Educação, Secretaria de Educação Especial, Secretaria de Educação a Distância, 2007.

SCHAFFAZICK, SR et al. Caracterização e estabilidade físico-química de sistemas poliméricos nanoparticulados para administração de fármacos. In: Química Nova, v. 26, n. 5, 2003. p. 726-737.

SILVA, GA. Introduction to nanotechnology and its applications to medicine. In: Surgical Neurology, v.61, 2004. p.216-220.

UFRGS. INCLUIR - Núcleo de Inclusão e Acessibilidade. Disponível em: <http://www.uf rgs.br/incluir>. Acesso em: 30 mai. 2016.

WANG, $S$ et al. Application of nanotechnology in improving bioavailability and bioactivity of diet-derived phytochemicals. In: Journal of Nutritional Biochemistry, $n$. 25, 2014. p. 363-376.

ZABALA, A. A prática educativa: como ensinar. Porto Alegre: Artmed, 1998. 